\title{
On-Site Efficiency Analysis of a Generator in the Millisecond Range
}

\author{
Bas ten Have \\ University of Twente \\ Enschede, The Netherlands \\ bas.tenhave@utwente.nl
}

\author{
Niek Moonen \\ University of Twente \\ Enschede, The Netherlands
}

\author{
Frank Leferink \\ University of Twente, Enschede \\ THALES Nederland B.V., Hengelo \\ The Netherlands
}

\begin{abstract}
Power supply systems, such as a diesel generator, are often designed based on the apparent power and the peak demands of non-linear loads. Because of this the generators run on a lower percentage of the rated load for most of their operation hours and are thus overdimensioned. The efficiency of generators is very poor when these are not running on full power. So, optimizing the power system such that it runs on a higher percentage of the rated load makes the system more efficient. In this paper measurement techniques used to determine the efficiency are researched. A system that uses a diesel generator is measured on-site, and analyzed using conventional power quality measurements and time-domain measurements in the millisecond range. The aim is to get more detailed insight in the behavior of the system and optimize the power supply system accordingly.

Index Terms-Efficiency, Generator, Non-linear loads, Power Quality, Time-domain measurements
\end{abstract}

\section{INTRODUCTION}

When designing a power supply system the apparent power is taken into account. This includes the reactive power, which is not consumed by the load, but this power needs to be supplied by the power system. In [1] an example is given of a building that has a real power consumption below $3 \mathrm{MW}$, but due to all the harmonic distortion generated by non-linear devices, the buildings power supply is overdimensioned at 7.2 MVA, based on the apparent power. This is more than 2 times higher than the active power, and results in high costs for the needed power supply system.

Non-linear behavior of modern electronic equipment can generate electromagnetic interference (EMI), which can generate malfunctioning of devices or systems [2]. The nonlinear effects of these loads are in the millisecond range, as was showed in [3], were non-linear electronic devices largely affected static energy meters, these meters are used to measure the energy consumption in residential situations for billing purposes.

Furthermore, large consuming loads cause peaks in the energy demand, and power systems are designed to these peak demands. As a result the supply runs on a smaller percentage of these peak demands, and thus of its rated nominal power, in most of its operating hours. In industry many generators used

This project has received funding from the EMPIR programme co-financed by the Participating States and from the European Union's Horizon 2020 research and innovation programme. The results found reflect the author's view only. EURAMET is not responsible for any use that may be made of the information it contains. do not exceed $20 \%$ of the rated load [4]. Diesel generators are often used as power supply in micro grids, and the efficiency of diesel generators is very poor when these are not running on full operation power [5].

The effect of current distortion on a weak supply grid was investigated in [6], it was shown that for low power loads connected to the generator, the supplied voltage waveform was heavily distorted, resulting in high harmonic distortion. Switching multiple loads on simultaneous, resulted in high inrush currents causing stability problems of the generator. These high peak values could result in a crash of the generator, resulting in a complete shutdown of the system, causing potentially serious problems for the users.

Optimizing the power system to have a diesel generator that is used more efficiently could result in serious fuel savings and a less distortion [7]. This may require super-capacitors, to cope with the peak demands [8], or filter techniques to create a better adaption between load and supply.

This paper researches measurement techniques that can be used to determine the efficiency of a power system, taking the aforementioned issues into account, and optimizing the system accordingly. This is done by analyzing a system that uses a diesel generator. The measurements are performed on-site, so when the system is operational, aimed to investigate whether the system is designed properly and if the rated nominal power of the diesel generator should be redesigned. An analysis is made using conventional power quality measurements over a large interval, followed by a more detailed analysis in the millisecond range. The millisecond analysis was performed at the moments that the system was switched between normal and heavy loading states. This shows the behavior of the system when these peak values occur, and can determine the duration and repetition of the peaks, or the running active power. This analysis can give more detailed insights compared to the conventional power quality analysis, and could help when optimizing the system to get a more efficient generator.

The rest of this paper is organized as follows: in Section II the measurement method is described, first the system under test is described briefly, then both the conventional power quality and millisecond measurement techniques are introduced. Section III describes measurement results obtained using both techniques. Then in Section IV the both methods are discussed. Finally, in Section V a conclusion is made about 
the efficiency of the system under test and it is concluded that the introduced measurements in the millisecond give valuable additional results for the optimization of the system.

\section{METHOD}

In this section first the system under test is introduced, followed by the method used in conventional power quality measurements, and finally a measurement technique is introduced which measures the system in the millisecond range.

\section{A. System under test}

The system under test is supplied by a $40 \mathrm{~kW}$ motor generator, used to power two parts of the system, Fig. 1. The bottom part of the system consist of the secondary loads, these contain the cooling systems, motor control unit and temperature control unit. The top part consist of a transformer and the primary load of the system. This load does not run on full power, but will draw more power in intervals. Therefore, determining the generated peak demands (of this primary load) is of great importance when optimizing the system.

In Fig. 1 two measurement positions are indicated: position 1 is directly after the generator and covers the complete system, and position 2 covers the primary load before the transformer, so excluding the secondary loads. As this load will generate high peak demands, it is beneficial to measure it when excluding the secondary loads.

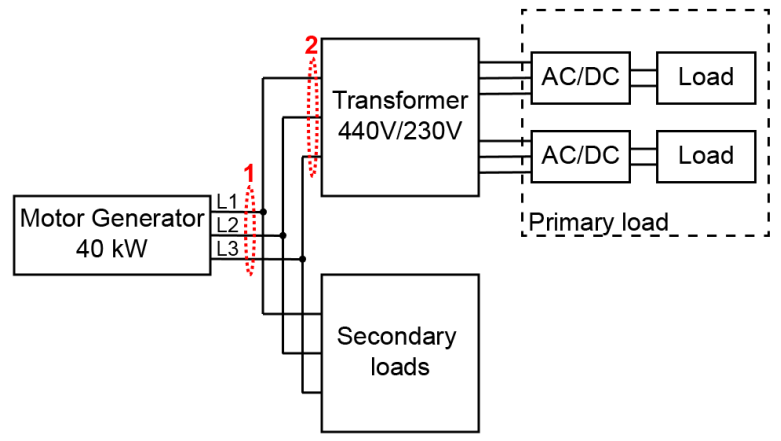

Fig. 1: Schematic of the system under test.

\section{B. Power quality method}

Power quality measurements are performed according the IEC 61000-4-30 [9]. These measurements are performed at position 1 and 2 as indicated in Fig. 1.

This is done using a power quality analyzer model WT 500 from Yokogawa. The currents are measured using non-invasive current transducers (CTs) model TA167 from Pico Technology TA167 CTs, and the range of the CTs is set to $200 \mathrm{~A}$.

The following parameters are determined during these measurements: the root mean square values of the voltage
$\left(U_{r m s}\right)$ and current $\left(I_{r m s}\right)$, the real $(P)$, apparent $(Q)$, and reactive power $(S)$, the power factor $(P F)$, the minimum $\left(I_{\min }\right)$, maximum $\left(I_{\max }\right)$, and crest factor $\left(C f_{I}\right)$ of the current.

\section{Millisecond measurement technique}

Additional time-domain measurements are done in the millisecond range. These are only performed at position 2, Fig. 1, as this load will introduce the peak demands of the system.

This measurement is performed using the same CTs as mentioned before, passive 1:100 voltage probes, and a 5444D Picoscope digitizer from Pico Technology.

By using this measurement method the voltage and current will be captured in the millisecond range, so on waveform level. From this data, a running average power per cycle of the waveform can be determined, this gives detailed information about the occurrence and duration of the peaks introduced by the system. Furthermore, the amplitude probability distribution (APD) function can be used to determine probability that the current amplitude reaches a certain level [10], [11].

\section{RESULTS}

In this section, first the contribution of the complete system is measured using the power quality method. Secondly, the contribution of the primary load is determined using both power quality and millisecond range measurements.

\section{A. Contribution of complete system}

To study the power consumption and the peak demands occurring in the complete system when operational, power quality measurements are performed. Table I shows the maximum values that occur per measurement interval. The apparent power $(S)$ is 1.7 times higher than the active power $(P)$, which is significant. The average active power per second is shown in Fig. 2, the system goes from stand-by to operational after 60 seconds and shut down after 320 seconds. The power consumption fluctuates a bit, and a peak is clearly visible when the system is turned on. After startup a power sag is noted, at $110 \mathrm{~s}$, which could be a measurement error. No other high peaks in the average power are observed.

\section{B. Contribution of the primary load}

Now, the contribution of the primary load to the system is measured. The power quality is measured and extreme values that occur per measurement interval are shown in Table II. For the current on L1 a minimum value $\left(I_{\min }\right)$ of $-112 \mathrm{~A}$ is found, resulting in a crest factor $\left(C f_{I}\right)$ of 7.3 , this value is quite high compared to the other minimum and maximum values found, additional measurements are required to verify this minimum. During the measurements the loads operation mode is changed three times, between stand-by and fully operating. The average

TABLE I: Results of the power quality measurements of the complete system, measurement position 1 .

\begin{tabular}{|r|r|r|r|r|r|r|r|r|r|}
\hline & $U_{r m s}(\mathrm{~V})$ & $I_{r m s}(\mathrm{~A})$ & $I_{\max }(\mathrm{A})$ & $I_{\min }(\mathrm{A})$ & $P(\mathrm{~W})$ & $S(\mathrm{~W})$ & $Q(\mathrm{~W})$ & $P F$ & $C f_{I}$ \\
\hline L1 & 399 & 26 & 95 & -100 & 15242 & 17068 & 8043 & 0.85 & 3.6 \\
L2 & 399 & 28 & 115 & -111 & 7990 & 18006 & 16136 & 0.41 & 4.2 \\
L3 & 399 & 27 & 58 & -71 & 5393 & 12636 & 11695 & 0.45 & 2.1 \\
Total & & & & & 28625 & 47710 & & & \\
\hline
\end{tabular}




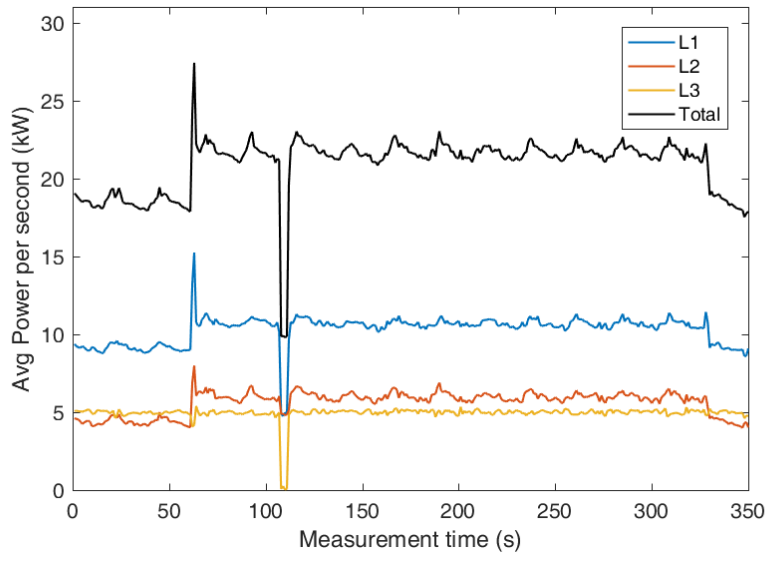

Fig. 2: Power quality measurement of average real power per second at generator, position 1 .

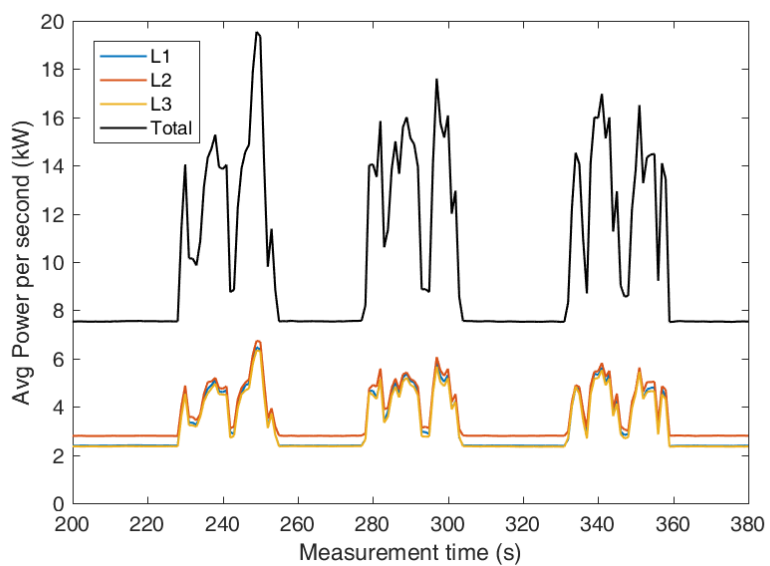

Fig. 3: Power quality measurement of average real power per second of primary load, position 2 .

active power per second is plotted in Fig 3. It is observed that the power is not constant when the load is in operation mode.

To analyse the behavior in more detail, time-domain measurements in the millisecond range are performed. The current waveforms show a distorted sinusoid, that has two peaks in this time interval, Fig 4. After the peak there is a dip in the current amplitude for a short time instance. The negative peak current on L1, as was found using the power quality analysis, was not found using this time-domain measurement. As Table III shows the extreme current values found in this analysis. Except from this peak the other values show similar results as in the power quality analysis.

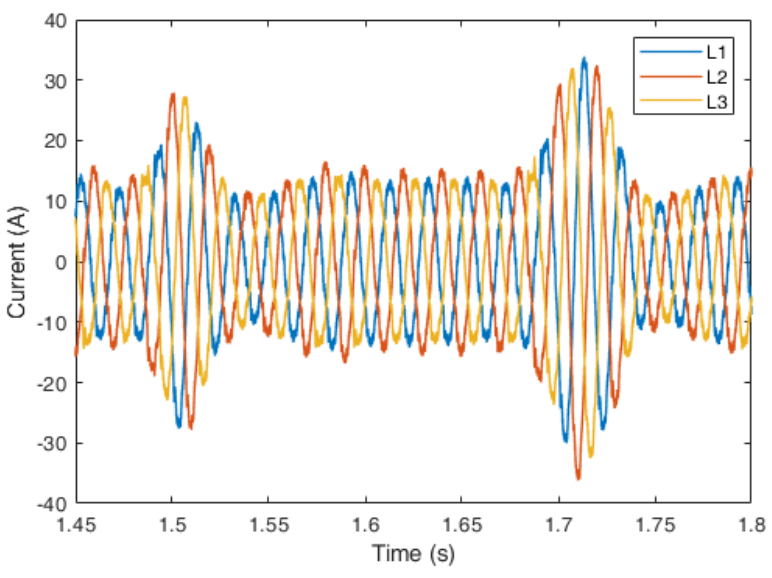

Fig. 4: Snapshot of the time-domain behavior of the current at the primary load, position 2 , using time-domain measurement.
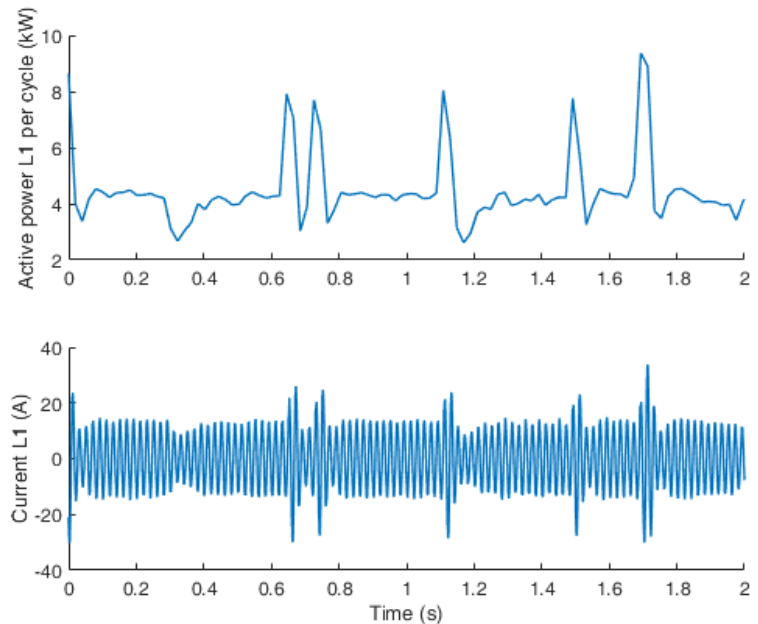

Fig. 5: Active power on L1 per cycle and line-current at the primary load, position 2 , using time-domain measurement.

Because the L1 current showed unexpected minima before, it was further analyzed. The active power per cycle was calculated, Fig. 5, this is an enlargement of Fig. 4. It shows peaks with a duration of only one or two periods, furthermore it can be seen that peaks occur quite often, as between $0.6 \mathrm{~s}$ and $0.8 \mathrm{~s}$ already two peaks occur. This makes optimization using super-capacitors more difficult, as the capacitor has limited time to charge. From Fig. 5, maximum active power values up to $8 \mathrm{~kW}$ are found which is slightly higher than using the power quality analysis, Fig. 3.

TABLE II: Results of the power quality measurements of the primary load, measurement position 2 .

\begin{tabular}{|r|r|r|r|r|r|r|r|r|r|}
\hline & $U_{r m s}(\mathrm{~V})$ & $I_{r m s}(\mathrm{~A})$ & $I_{\max }(\mathrm{A})$ & $I_{\min }(\mathrm{A})$ & $P(\mathrm{~W})$ & $S(\mathrm{~W})$ & $Q(\mathrm{~W})$ & $P F$ & $C f_{I}$ \\
\hline L1 & 383 & 13 & 39 & -112 & 6593 & 7782 & 4780 & 0.86 & 7.3 \\
L2 & 386 & 13 & 38 & -41 & 6921 & 7929 & 4099 & 0.88 & 2.9 \\
L3 & 384 & 12 & 36 & -45 & 7020 & 8210 & 1341 & 0.85 & 3.0 \\
Total & & & & & 20534 & 23921 & & & \\
\hline
\end{tabular}


For the currents on all lines, as captured during the millisecond range measurements, the APD is determined in Fig. 6. The root mean square of all currents is around $10 \mathrm{~A}$, Table III, and from the APD diagram it is clear that it is still quite likely that currents with a higher amplitude occur, as $10 \mathrm{~A}$ has a probability of 0.4 . Whereas, it is unlikely to have values above $25 \mathrm{~A}$, as the probability is then below 0.01. Furthermore, it is more likely to have higher amplitudes on L2 compared to L1 and L3.

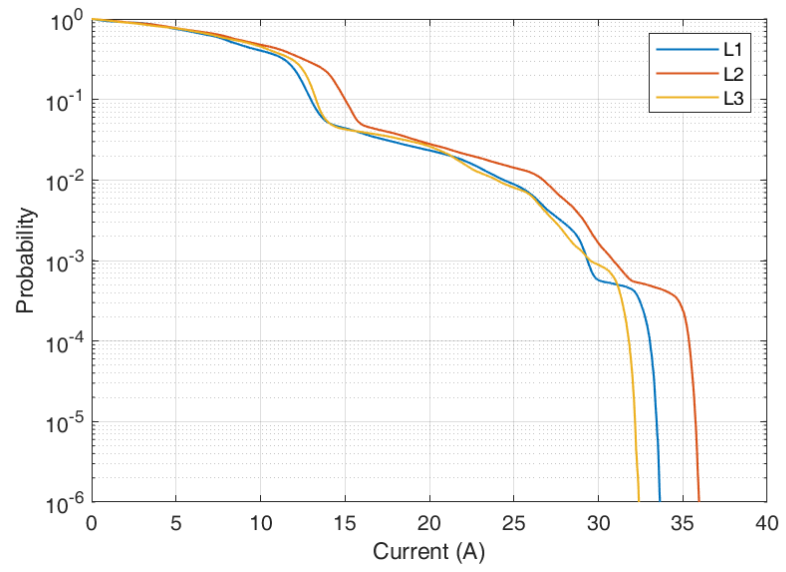

Fig. 6: Amplitude probability distribution of the currents on all lines, obtained from the millisecond range measurement.

TABLE III: Analysis of current using millisecond range technique at the primary load, position 2 .

\begin{tabular}{|l|r|r|r|r|}
\hline & $I_{r m s}(\mathrm{~A})$ & $I_{p k \max }(\mathrm{A})$ & $I_{p k m i n}(\mathrm{~A})$ & $C f_{I}$ \\
\hline L1 & 10 & 34 & -33 & 3.5 \\
L2 & 11 & 32 & -37 & 3.6 \\
L3 & 10 & 33 & -33 & 3.3 \\
\hline
\end{tabular}

\section{Discussion}

The introduced measurements in the millisecond range give additional information for the optimization of the system, compared to the conventional power quality measurements. As it gives information about the occurrence of the peak values in a small millisecond range. Furthermore, additional information such as the APD diagram can be obtained.

Next to this, the method can be used to determine the same parameters as during conventional power quality measurements. However, there is a difference in between the values of the parameters in the shown measurements. This can be explained because the measurement interval of the performed millisecond range measurements was significant lower than the power quality measurements. But with a similar measurement interval and decent post processing all of the parameters found using the power quality method could also be extracted using the millisecond range measurements.

\section{CONCLUSION}

It was shown that monitoring the peak values of a system in a short millisecond range can give better insights compared to conventional power quality analysis methods. The peaks can be visualized more clearly and it can be determined how often and how long the occurrence is. This can help in optimizing the design, for example by using super-capacitors or active filtering techniques to cope with the peak demands. Next to this, all the information retrieved from the power quality method can also be obtained from the millisecond range measurements.

For the system under test the power drawn by the loads will not form an issue for the generator. However, a strange peak value of $-112 \mathrm{~A}$ on $\mathrm{L} 1$ at the primary side of the transformer was not explained. The high reactive power on L2 and L3 found when measuring the complete system can result from one of the sub-parts in the system that is not measured now, as the measurements at the primary load did not show this high reactive power.

Future measurements should be done using similar measurements in the millisecond range, but should be performed at multiple measurement points within the system. Such that all the sub parts in the system are measured at the same time, to monitor how the sub parts affect each other. This should be combined with a power quality analysis of the complete system. This could give a more complete picture of the system and can help in optimizing the system even further.

\section{REFERENCES}

[1] R. B. Timens, F. J. Buesink, V. Ćuk, J. F. Cobben, W. L. Kling, and F. Leferink, "High harmonic distortion in a new building due to a multitude of electronic equipment," IEEE International Symposium on Electromagnetic Compatibility, pp. 393-398, 2011.

[2] F. Leferink, "Conducted interference, challenges and interference cases," IEEE Electromagnetic Compatibility Magazine, vol. 4, no. 1, pp. 78-85, 2015.

[3] B. Have, T. Hartman, N. Moonen, and F. Leferink, "Inclination of Fast Changing Currents Effect the Readings of Static Energy Meters," 2019 International Symposium on Electromagnetic Compatibility (EMC Europe 2019), pp. 208-213, 2019.

[4] O. Kurka and J. Leuchter, "New generation of mobile electrical power sources," Proc. of the Int. Conf. on Electrical Machines (ICEM 2000), 2000.

[5] M. Greig and J. Wang, "Fuel Consumption Minimization of VariableSpeed Wound Rotor Diesel Generators," in IECON 2017 - 43rd Annual Conference of the IEEE Industrial Electronics Society, 2017.

[6] R. B. Timens, F. J. Buesink, and F. Leferink, "Effect of energy saving lights on power supply," IEEE International Symposium on Electromagnetic Compatibility, pp. 8-11, 2012.

[7] H. Hilal, M. A. Oktaufik, A. Prastawa, B. Prasetyo, and R. Hutahaean, "Smart Diesel Generator to compensate on-grid PV fluctuation: A case study in Sumba Island Indonesia," 3rd IEEE Conference on Power Engineering and Renewable Energy, ICPERE 2016, pp. 33-37, 2017.

[8] D. A. Permana, U. Sholahuddin, D. Hamdani, and T. D. Rachmildha, "Study of Supercapacitor Utilization on Regenerative Braking System: Design and Simulation," Proceeding - 2018 5th International Conference on Electric Vehicular Technology, ICEVT 2018, pp. 88-94, 2019.

[9] "Electromagnetic compatibility (EMC) - Part 4-30: Testing and measurement techniques - Power quality measurement methods," IEC 6100-4-30 Ed. 3, 2012.

[10] "Specification for radio disturbance and immunity measuring apparatus and methods - Part 1-1: Radio disturbance and immunity measuring apparatus - Measuring apparatus," CISPR 16-1-1, 2015.

[11] M. Pous, M. A. Azpúrua, and F. Silva, "APD oudoors time-domain measurements for impulsive noise characterization," 2017 International Symposium on Electromagnetic Compatibility - EMC EUROPE 2017, EMC Europe 2017, 2017. 